\title{
The Failure Criterion of Single-Layer Spherical Lattice Shell Based on Kinetic Energy
}

\author{
Panfeng Ba, Yigang Zhang, Jinzhi Wu, and Zhihao Zhang \\ Spatial Structures Research Center, Beijing University of Technology, Beijing 100124, China \\ Correspondence should be addressed to Panfeng Ba; bapanfeng@emails.bjut.edu.cn
}

Received 25 April 2015; Accepted 7 July 2015

Academic Editor: Dapeng P. Du

Copyright (C) 2015 Panfeng Ba et al. This is an open access article distributed under the Creative Commons Attribution License, which permits unrestricted use, distribution, and reproduction in any medium, provided the original work is properly cited.

\begin{abstract}
The dynamic failure criterion of single-layer spherical lattice shells has been an important research subject. The paper examines dynamic failures of single-layer spherical lattice shells and proposes the structure dynamic failure criterion based on the kinetic energy. The failure criterion was demonstrated through the dynamic failure test on a single-layer spherical lattice shell. Then, simulation analysis was carried out through two cases with material damage taken into account. The proposed failure criterion can accurately identify failure moments caused either by strength fracture or by stability fracture.
\end{abstract}

\section{Introduction}

Single-layer spherical lattice shell is highly favored in practice for its light weight and graceful appearance by experts and scholars. The recent high frequency of earthquakes has challenged the extensive application of this light-weighted shell. Thus, the dynamic failure of the single-layer spherical lattice shell has been considered as an important research subject. The intensity fracture and stability fracture were extensively identified by experts as the major dynamic failures of singlelayer spherical lattice shell. Shen et al. [1-3] identified that dynamic strength failure occurs when the ratio of plastic bar is greater than $42 \%$ and maximum node displacement exceeds span 1/100 in the K8 single-layer spherical lattice shell from an overall perspective of integral structure. Du et al. [4] established a double-control principle based on plasticity dissipated energy and the ultimate displacement, which considers the failure of integral structure caused by damage accumulation and thus could identify the strength fracture and stability fracture. Nevertheless, Du's principle could not go further than providing evaluation of degree of damage. Zhang and Peil [5] defined the stability concept and proposed a method identifying the stability of integral structure by changes in stability, considering the total potential energy of the rod and the ratio between increment in structural strain energy and potential energy. Nevertheless, Zhang's method is limited to the elasticity problem.

In theory, failure mechanism of single-layer spherical lattice shell and determining the structure of the ultimate load are important subject with maximum displacement and the degree of plastic as a major focus. Despite being highly valued in engineering application, the effective preestimating method of the failure of single-layer for the spherical lattice shell which could be applied to improve structure failureresisting capacity by strengthening vulnerable spots is yet to be further explored. The authors resort to detecting a macroscopic quantity as a preestimating failure criterion which is easy to calculate and also reflects the characteristics of the overall structural failure. The authors note that there must be vibration and dramatic changes in kinetic energy in dynamic failures. According to the findings stated above, the paper endeavors to propose the dynamic failure criterion based on the kinetic energy and explores whether there exists a failure criterion coefficient applicable to identify dynamic failure and failure moment under strong shock. Moreover, the paper also sets out to verify the validity of the proposed failure criterion coefficient by referring to the collapse test data and the case which stimulates the process of the collapse of singlelayer spherical lattice shells. 


\section{The Dynamic Failure Criterion of Single-Layer Spherical Lattice Shells}

In finite element calculation, the kinetic energy equation can be written as follows:

$$
E_{k}(t)=\frac{1}{2}\{\dot{U}(t)\}^{T}[M]\{\dot{U}(t)\},
$$

where $E_{k}(t)$ is kinetic energy of whole structure, $[M]$ is the mass matrix of structure, $\{U(t)\}$ is the displacement of matrix, and $\{\dot{U}(t)\}$ is the first derivative. During any arbitrary periods $[t, t+\Delta t]$, the increment in the kinetic energy can be expressed as follows:

$$
\begin{aligned}
\Delta E_{k}= & E_{k}(t+\Delta t)-E_{k}(t) \\
= & \frac{1}{2}\{\dot{U}(t+\Delta t)\}^{T}[M]\{\dot{U}(t+\Delta t)\} \\
& -\frac{1}{2}\{\dot{U}(t)\}^{T}[M]\{\dot{U}(t)\} .
\end{aligned}
$$

$\Delta E_{k}$ is the increment of kinetic energy. The acceleration at any period can be expressed as $\ddot{U}(\beta)$ as follows:

$$
\{\dot{U}(t+\Delta t)\}=\{\dot{U}(t)\}+\ddot{U}(\beta) \Delta t .
$$

$\{\ddot{U}(\beta)\}$ is the second derivative. Substituting (3) into (2), we can get the following:

$$
\begin{aligned}
\Delta E_{k} & \frac{1}{2}\{\{\dot{U}(t)\}+\ddot{U}(\beta) \Delta t\}^{T}[M]\{\{\dot{U}(t)\}+\ddot{U}(\beta) \Delta t\} \\
& -\frac{1}{2}\{\dot{U}(t)\}^{T}[M]\{\dot{U}(t)\} \\
= & \frac{1}{2}\{\dot{U}(t)\}^{T}[M]\{\dot{U}(t)\} \\
& +\frac{1}{2}\left\{\{\dot{U}(t)\}^{T}[M]\{\ddot{U}(\beta)\}\right\} \Delta t \\
& +\frac{1}{2}\{\ddot{U}(\beta)\}^{T}[M]\{\dot{U}(t)\} \Delta t \\
& +\frac{1}{2}\{\ddot{U}(\beta)\}^{T}[M]\{\ddot{U}(\beta)\} \Delta t^{2} \\
& -\frac{1}{2}\{\dot{U}(t)\}^{T}[M]\{\dot{U}(t)\} .
\end{aligned}
$$

The single-layer spherical reticulated shell is a symmetric structure. The mass matrix of single-layer spherical lattice shell is symmetric matrices. An equation could be obtained: $[M]=[M]^{T}$, where $p$ is a constant in this equation. Then, anther equation can be written as follows:

$$
\begin{aligned}
\{\dot{U}(t)\}^{T}[M]\{\ddot{U}(\beta)\} & =p=p^{T} \\
& =\left[\{\dot{U}(t)\}^{T}[M]\{\ddot{U}(\beta)\}\right]^{T} \\
& =\{\ddot{U}(\beta)\}[M]\{\dot{U}(t)\}^{T} .
\end{aligned}
$$

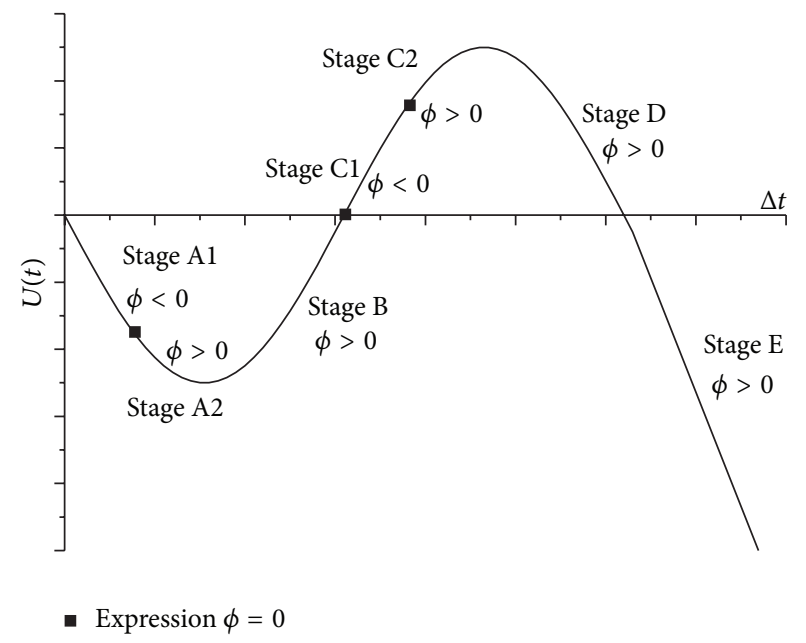

FIGURE 1: The relationship between displacement and coefficient.

Substituting (5) into (4), we can get

$$
\begin{aligned}
\Delta E_{k}= & \{\ddot{U}(\beta)\}^{T}[M]\{\dot{U}(t)\} \Delta t \\
& +\frac{1}{2}\{\ddot{U}(\beta)\}^{T}[M]\{\ddot{U}(\beta)\} \Delta t^{2} .
\end{aligned}
$$

Because $\Delta t>0$, when both sides of (6) divided by the time variable of $\Delta t$, we can get constant $\phi$ :

$$
\begin{aligned}
\phi= & \frac{\Delta E_{k}}{\Delta t} \\
= & \{\ddot{U}(\beta)\}^{T}[M]\{\dot{U}(t)\} \\
& +\frac{1}{2}\{\ddot{U}(\beta)\}^{T}[M]\{\ddot{U}(\beta)\} \Delta t .
\end{aligned}
$$

To be clear in discussion, several sign conventions could be stipulated as follows. The upward movement deviating from balance shaft is considered as positive and downward movement away from balance shaft as negative. Signs of node displacement, velocity, and acceleration velocity are in accordance with the above sign convention. The structure vibrates along the balance shaft. Vibration of situations could be vividly demonstrated in trajectory function $U(t)$ as stages of A, B, C, D, and E in Figure 1.

At Stage A1, the structure vibrates downward the balance shaft and the trajectory function $U(t)$ demonstrates that most particles moved downward away from the balance shaft. The speed is larger; the restoring force causes acceleration from zero to positive. So $\dot{U}(t)<0, \ddot{U}(\beta)>0$, and the coefficient $\phi$ is greater than zero at the multiple consecutive time $\Delta t$.

When the movement was between Stages A1 and A2 and $|\dot{U}(t)|=(1 / 2)|\ddot{U}(\beta)| \Delta t$, the coefficient $\phi=0$, which indicates kinetic energy increment, was zero.

When the movement reaches Stage A2 and $\dot{U}(t)<0$, $\ddot{U}(\beta)>0$, the trajectory function $U(t)$ demonstrates that most of the particles moved downwards deviating from 
the balance shaft. Nevertheless, $|\dot{U}(t)|<(1 / 2)|\ddot{U}(\beta)| \Delta t$ was still applicable in this stage and the coefficient $\phi$ is greater than zero at the multiple consecutive time $\Delta t$.

At Stage B and when $\dot{U}(t)>0, \ddot{U}(\beta)>0$, trajectory function $U(t)$ expresses most of the particles deviated from the balance shaft upward. The acceleration direction is in accordance with the previous stage, while the velocity direction changed. In other words, $\phi$ was greater than zero at the multiple consecutive time $\Delta t$.

By such analogy, the changing rule of coefficient $\phi$ in Stages C and D can be expressed as shown in Figure 1.

At Stage E, the global vibration of the structure begins continuous downward accelerated movement from zero. During the process, the accelerated speed direction changed, $\dot{U}(t)<0$, and $\ddot{U}(\beta)<0$. Moreover, signs of the acceleration and velocity are in accordance with each other at this stage.

The above discussion expresses that the coefficient $\phi$ contained the movement characteristics of structure. Relative to the static equilibrium state, the particle trajectory can be reflected according to the velocity and acceleration of symbols. The symbols of speed and acceleration determined the symbols of coefficient $\phi$ at the same time.

It can be noted in Figure 1 from phases $\mathrm{D}$ to $\mathrm{E}$ that, during multiple continuous $\Delta t, \phi>0$, which will cause continuous increase in speed. It can be also noticed that as both the value of $\phi$ and the kinetic energy of the structure escalate sharply, the corresponding structural displacement will continuously increase. Thus, it is fairly reasonable to identify the structural failure when the excessive structural displacement eventually causes the structural kinetic failure. Therefore, the previously last corresponding moment when $\phi=0$ can be recognized as the structural failure moment, which indicates the structure entered the failure state.

From what stated above, conclusions could be made that when the coefficient $\phi$ remains positive during minor continuous multiple time increments and the recorded maximum $\phi$ value $\left(\phi_{t=a}\right)$ is much larger than all the previously recorded $\phi$ values, the structural failure occurred. Correspondingly, during continuous increase of the $\phi$ value till $\phi_{t=a}$, the failure moment occurred when the $\phi$ value approaches zero from negative. Therefore, the coefficient $\phi$ could be considered as a failure criterion coefficient.

\section{Table Test Verification}

3.1. Introduction to Table Test. Combined with the shell tests conducted by scholars at home and abroad [6-10], the shaking table experiment adopts the single-layer spherical lattice shell model. The span is $3 \mathrm{~m}$ and there are 4 loops. The rise-span ratio is $1: 5$ and the stress bars are the steel pipes whose diameters are $\varnothing 10 \times 1 \mathrm{~mm}$ or $\varnothing 8 \times 1 \mathrm{~mm}$ as shown in Figure 2. Solid spheres are installed on spherical joints, with each sphere having a diameter of $200 \mathrm{~mm}$ and weighing $32.4 \mathrm{~kg}$, as shown in Figure 3 . Considering the selfvibration characteristics of test model, we used the vertical harmonic load to enhance vibration. Harmonic load is the basic component of dynamic load and all kinds of complicated earthquake loads can be divided into combinations
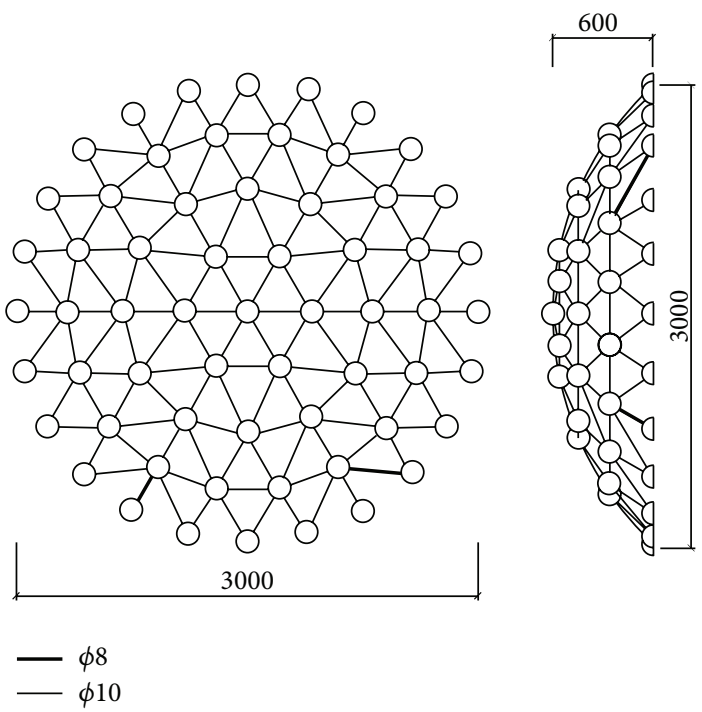

FIgURE 2: The size of test model.

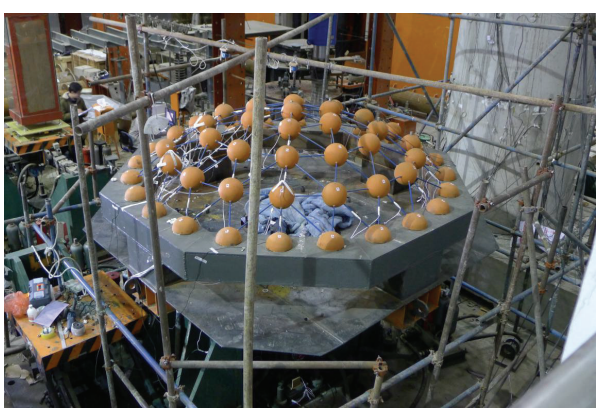

Figure 3: The real test model.

of harmonic load with different frequencies and amplitudes. Consequently, harmonic load is adopted in the test.

The test was conducted in the Structural Testing Center of Beijing University of Technology. In the test, we used two sets of noncontact displacement acquisition test which are produced by the company of IMETRUM in England. The test applied a tracking shot to record the displacement of all nodes. The process of collapse under the strong earthquake was shown in Figure 4.

3.2. Test Result Analysis. Particles' absolute displacement time history was calculated, with particles' displacement time history curve and the displacement input of bearings taken into consideration. Furthermore, the particle movement velocity history was obtained based on the derivation of discrete points and all particles' kinetic energy history. Then, the failure criterion coefficient $\phi$ of each moment $(\Delta t=$ $11 \mathrm{~ms}$ ) was calculated. The above procedures were conducted to make the program easier. It is observed that when $T=63.543 \mathrm{~s}$, the coefficient $\phi$ is less than zero. However, the failure criterion coefficient values are all greater than zero at three consecutive moments $(63.554 \mathrm{~s}, 63.566 \mathrm{~s}$, and $63.577 \mathrm{~s}$ ), which are, respectively, $7.004 \times 10^{5}, 1.437 \times 10^{6}$, and 


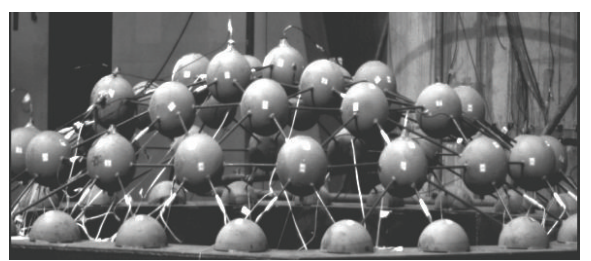

(a) $T=51.0 \mathrm{~s}$

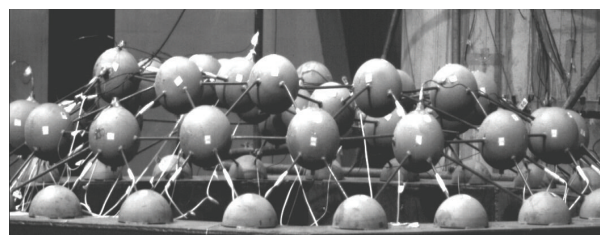

(c) $T=63.8 \mathrm{~s}$

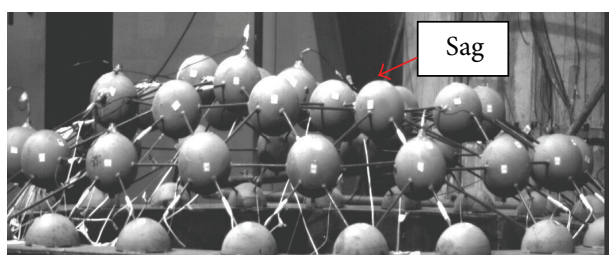

(b) $T=63.5 \mathrm{~s}$

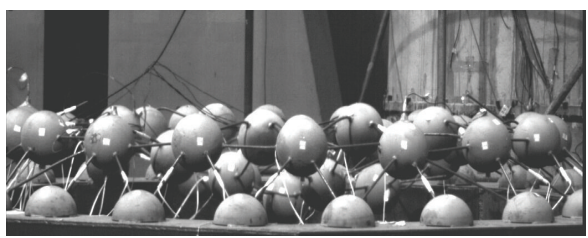

(d) $T=64.3 \mathrm{~s}$

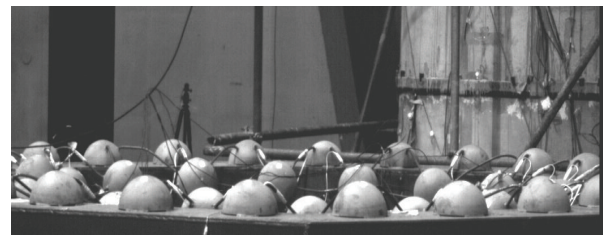

(e) $T=65.1 \mathrm{~s}$

FIgURE 4: The earthquake collapse process of test model.

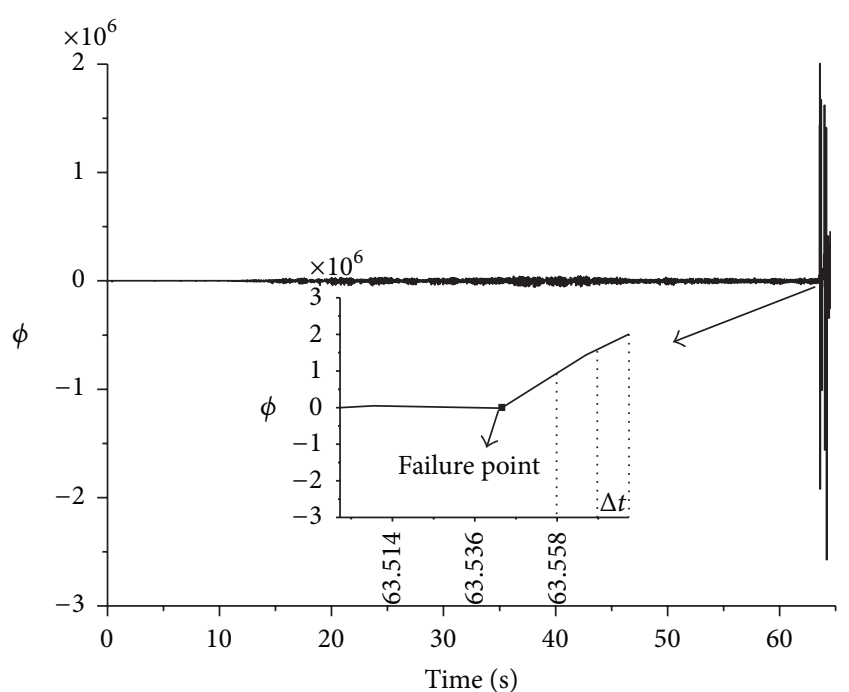

FIGURE 5: The test model discriminant coefficient curve of failure.

$2.007 \times 10^{6}$ and demonstrate a trend of increasing order of magnitude. The maximum $\phi_{t=a}=2.007 \times 10^{6}$ is much greater than the maximum $4.540 \times 10^{4}$ among all values of $\phi$ by $T=$ $63.544 \mathrm{~s}$. According to the criterion, the structure is prone to failure at this moment. Combining with the two values of $\phi$ of two integral points, the corresponding moment of $\phi=0$ is $63.545 \mathrm{~s}$ by linear differential method. Thus, it can be decided that $T=63.545 \mathrm{~s}$ is structural failure point.

Figure 5 shows the time history curve of the discriminant coefficient $\phi$ of the test model. It can be seen that coefficient

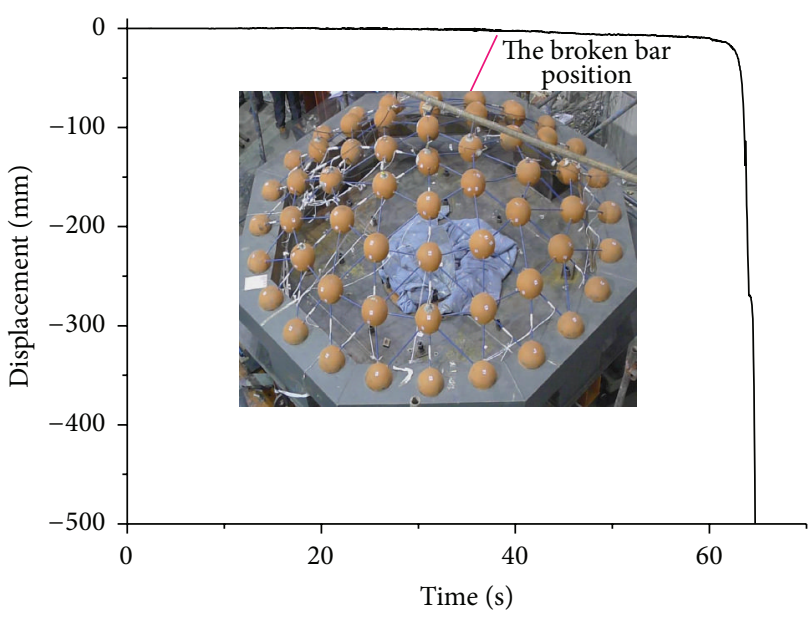

FIGURE 6: The center point displacement curve of test model.

$\phi$ fluctuates near the zero line before $63.554 \mathrm{~s}$ but increases sharply after $63.554 \mathrm{~s}$. It can also be observed from Figure 4(b) that, at the moment of $63.5 \mathrm{~s}$ in the test, an inward concave appeared in the test model resulting from the deviation of some joints from the equilibrium position. The sag develops from the net shell inner ring to the outer ring (Figure 4(c)). When $T=64.3 \mathrm{~s}$, the latticed shell collapsed. It shows that it is reasonable to identify $T=63.545 \mathrm{~s}$ as structural failure moment according to the failure criterion.

Figure 6 presents the center point displacement curve of test model. It can be observed that the displacement value of the center point fluctuates around a certain value. 

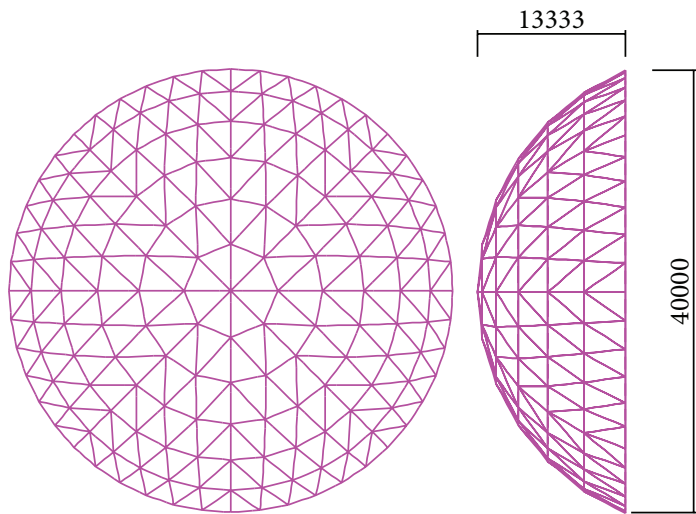

FIGURE 7: The shell model of paper [1].

At about $40 \mathrm{~s}$, there has been significant bar fracture when the center point displacement time history curve decreased slightly. Though the center point displacement is $3.6 \mathrm{~mm}$, the structure can still bear load and has not yet entered the failure state. At about $63 \mathrm{~s}$, the center point displacement sharply increased, prone to failure. It is a further test for verifying the criterion based on the kinetic energy which can accurately determine the exact time of structure failure.

\section{The First Calculation Model}

4.1. Model Data. The span of single-layer spherical lattice shell is $40 \mathrm{~m}$ in paper [1]. Rise-span ratio is $1 / 3$. All bar is hotrolled steel pipe of which the ribbed bar section is $\varnothing 114 \times 3.0$, $\varnothing 127 \times 3.5$, and $\varnothing 140 \times 4.5(\mathrm{~mm})$. The bearing hinge pedestal is assumed, roofing gravity load of $200 \mathrm{~kg} / \mathrm{m}^{2}$. The roofing gravity load will be concentrated onto the nodes. Rayleigh damping is used, with its damping factor as 0.02 . Finite element method first establishes unit stiffness matrices by dividing the bar into several units and then the whole stiffness matrix. The paper uses finite element software ABAQUS. The model of Figure 7 introduced UMAT subroutine that, considering material damage [10], the amplitude of the harmonic load is $100 \mathrm{gal}$. The load frequency is $2.6 \mathrm{~Hz}$.

Literature [1] concludes that when the load amplitude is 97 gal, there is neither significant local rigidity nor obvious displacement in vibration equilibrium position. Only $0.06 \%$ of the whole structure become plastic bars and little vertical displacement took place. When the load amplitude is $97 \mathrm{gal}$, the local destruction becomes obvious and rapidly develops till the collapse of the whole structure, which could be regarded as a typical example of stability failure. Literature [1] also presents the history curve of the whole structure displacement under different loads in Figure 8. According to Figure 8, the structure failure moment starts at about $5 \sim 6 \mathrm{~s}$.

4.2. Analysis of Calculation Results. The history curve of failure coefficient $\phi$ of the structure model was calculated under $100 \mathrm{Gal}$ seismic amplitude. When $T=2.305 \mathrm{~s}$, the recorded value of coefficient $\phi$ is $1.08 \times 10^{7}$. The failure coefficient $\phi$ was, respectively, $1.1 \times 10^{9}$ and $-4.48 \times 10^{8}$ at two

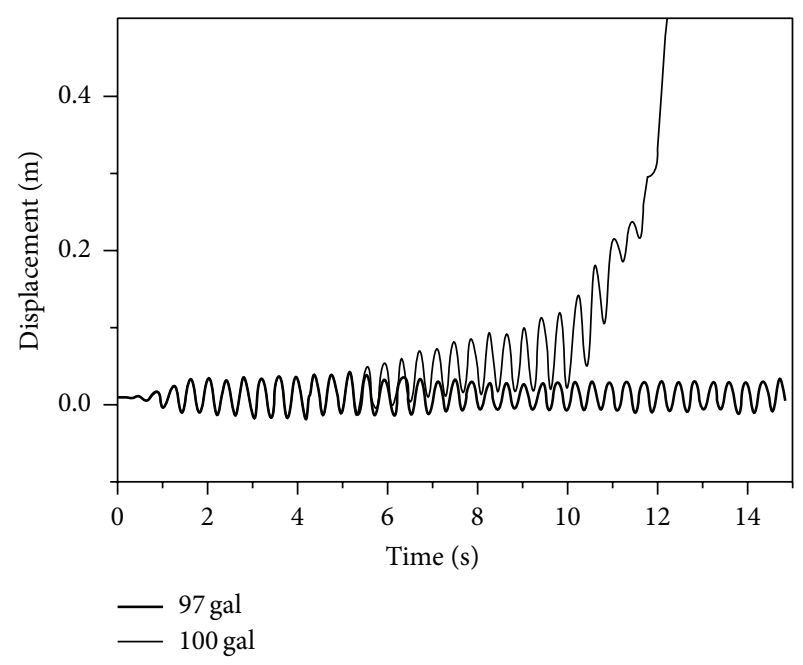

FIGURE 8: The displacement history curve of shell model.

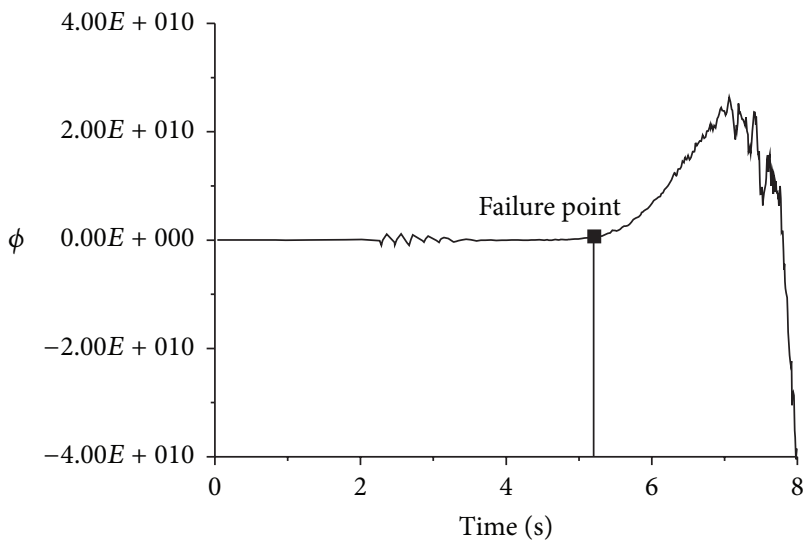

FIGURE 9: The history curve of failure coefficient.

other subsequent moments. These values, however, had yet not met the failure criterion and thus could be ignored.

Fluctuation developed according to the recorded history curve of coefficient $\phi$. At the time of $T=4.67 \mathrm{~s}$, the recorded value was $9.3 \times 10^{7}$. Then, the value of the coefficient remained positive and increased rapidly during 154 consecutive $\Delta t \approx$ $30 \mathrm{~ms}$. For the three different time points $(4.72 \mathrm{~s}, 5.45 \mathrm{~s}$, and $7.06 \mathrm{~s})$, the $\phi$ values were, respectively, $2.32 \times 10^{7}, 1.82 \times 10^{9}$, and $2.6 \times 10^{10}$, showing a sharply increasing trend. According to the failure criterion, $4.634 \mathrm{~s}$ was defined as the failure point of the overall structure through calculation by the linear difference method. As it can be seen from Figure 9, the structural failure moment has obvious characteristic which meets the failure criterion.

The failure moment $4.634 \mathrm{~s}$ is slightly ahead of the failure moment identified in literature [1]. This is mainly because this paper considered the material damage accumulation effect in the calculation. It also shows that the application coefficient $\phi$ can accurately distinguish the failure time of the structure in the dynamic stability failure. 

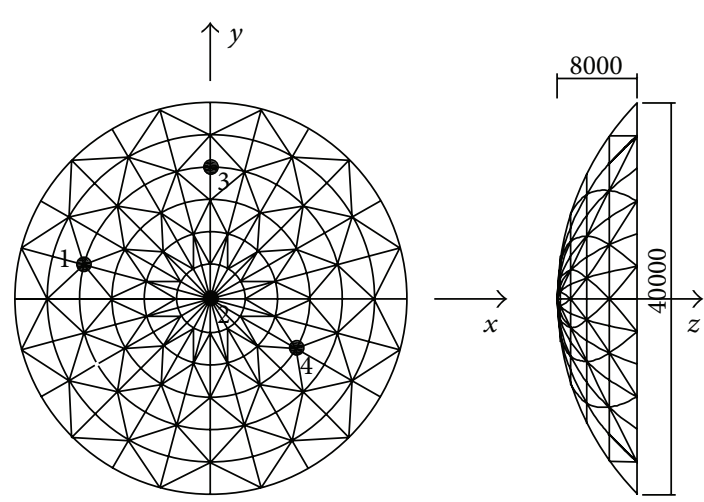

FIGURE 10: The diagram of overall structure.

\section{The Second Calculation Model}

5.1. Model Data. Figure 10 [11] demonstrates that a six-ring Schwedler single-layer spherical lattice shell across 40 meters span was established, with vector ratio of $1 / 5$, roof gravity load of $2.5 \mathrm{kN} / \mathrm{m}^{2}$, and all ground holds hinged. The software of $3 \mathrm{D} 3 \mathrm{~S}$ is used for reasonable design and calculation. All bars are hot-rolled steel pipe, of which the main ribbed bar section is $\varnothing 165 \times 5$ and the rest of the link rod and diagonal section are $\varnothing 140 \times 4$. All bars are divided into four units of equal length and each unit's cross section is divided into 8 fibers of equal length. Considering geometric nonlinearity, the material yield point is $235 \mathrm{MPa}$ and the initial elastic modulus is $2.06 \times 10^{5} \mathrm{MPa}$ with Poisson's ratio as 0.3 .

The UMAT subroutine is introduced into the model structure shown in Figure 10 to analyze seismic response, taking damage accumulation into account. Three-directional Northridge wave is input, of which the PGA of $X$-direction is $1300 \mathrm{Gal}$ and the seismic peak values of $Y$-direction and $Z$ direction are, respectively, 0.85 and 0.65 times of $X$ 's.

5.2. Result Analysis. The calculation result shows that when $T=3.68 \mathrm{~s}$, the structure's coefficient $\phi$ is $1.67 \times 10^{9}$, which is comparatively large. When $T=3.64 \mathrm{~s}$ and $3.75 \mathrm{~s}$, two different timing points before and after $3.68 \mathrm{~s}$, the corresponding failure coefficient $\phi$ is, respectively, $1.07 \times 10^{8}$ and $-1.82 \times 10^{9}$. According to the structure failure criterion, this fluctuation of coefficient $\phi$ does not result in the structure failure. The fact that only a few bars become plastic bars together with the partial sag caused the fluctuation of the failure coefficient $\phi$. Later, during a succession of over $60 \Delta t \approx 15 \mathrm{~ms}$, the $\phi$ values remain positive values and keeps an increasing trend. When $T=24.64 \mathrm{~s}, 25.24 \mathrm{~s}$, and $25.77 \mathrm{~s}$, the corresponding $\phi$ values are, respectively, $1.4 \times 10^{8}, 4.18 \times 10^{9}$, and $1.19 \times$ $10^{10}$. And eventually the obtained maximum coefficient is $1.51 \times 10^{10}$, which is much greater than the maximum value recorded before $T=24.64 \mathrm{~s}$. Considering the structure failure criterion and the method of linear difference, when $T=$ $24.61 \mathrm{~s}$, the failure coefficient is zero. Figure 11 demonstrates the oscillation curve of the failure criterion coefficient, from which the failure moment could be clearly observed.

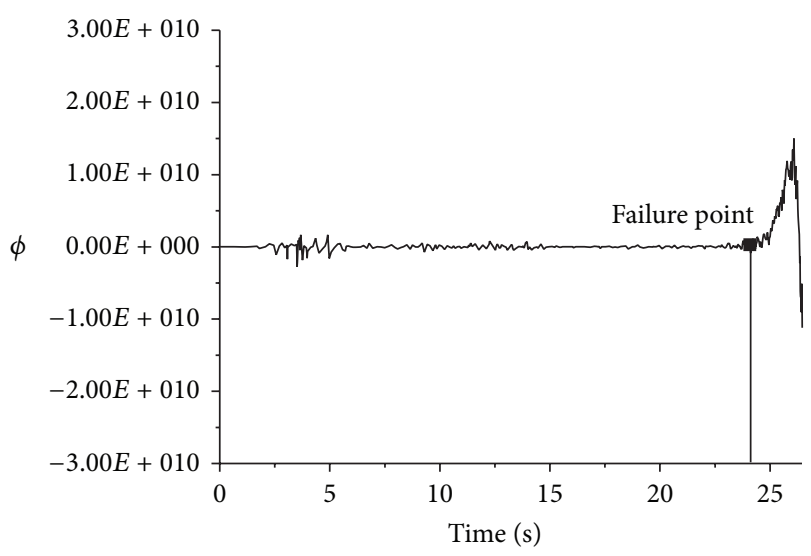

FIGURE 11: The history curve of coefficient $(\mathrm{PGA}=1300 \mathrm{Gal})$.

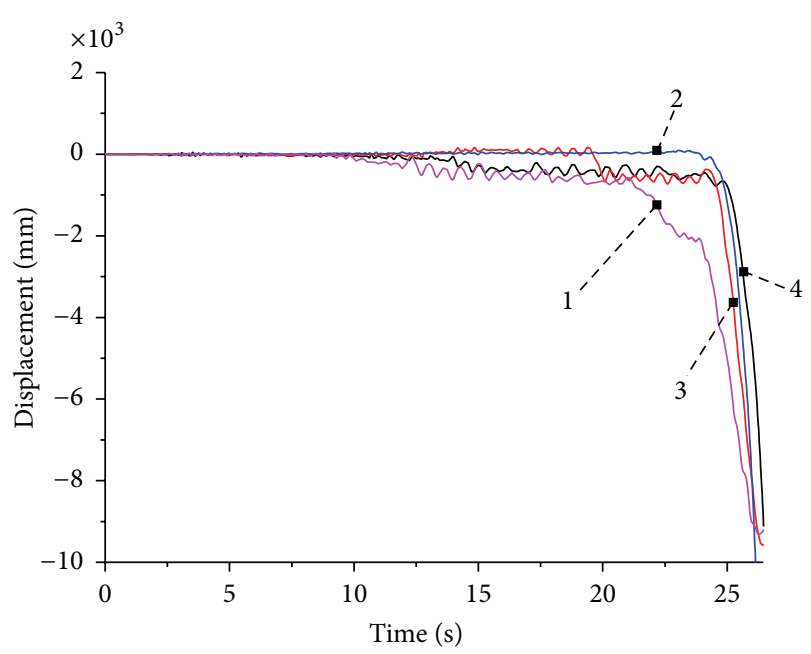

FIGURE 12: The displacement history curve of different point.

Figure 12 shows the time history curves of different node displacement. It can be observed in Figure 12 that moments when different joint displacement increases sharply are discrete. It is probably inadequate to identify the failure moment simply based on the displacement of the structure joints and might further require researchers' judgment. Especially when there is partial sag, this inadequate method might result in the misjudgment and mistakes. Contrastingly, the failure criterion used in this paper could be considered as adequate and reasonable.

The above analysis demonstrates that the vibration of the second calculation model becomes failure when $T=24.61 \mathrm{~s}$. Considering the mentioned criterion of failure moment, results show that $61.7 \%$ of bars in the structure have become plastic bars. Obviously, the damaged structure could serve as a typical example of dynamic strength fracture. Thus, a conclusion could be drawn that the failure criterion proposed in this paper can accurately identify the failure moment of the single-layer spherical lattice shells under dynamic strength fracture. 


\section{Conclusion}

In this study, the failure identification equation of single-layer spherical lattice shells is deduced based on kinetic energy. The dynamic failure criterion is verified by shaking table test data and two cases. Thus, several conclusions could be drawn as follows:

(1) The structure failure moment under dynamic loading can be identified based on the shift of failure criterion coefficient $\phi$ between positive or negative and its magnitude.

(2) The dynamic failure criterion based on kinetic energy is simple, practical, and thus easy to be programmed.

(3) The proposed failure criterion can accurately identify failure moment of single-layer spherical lattice shells damaged either by strength fracture or by stability fracture.

\section{Conflict of Interests}

The authors declare that there is no conflict of interests regarding the publication of this paper.

\section{Acknowledgments}

This work is partially supported by Natural Science Foundation of China under Grant nos. 51178009 and 91315301 and Beijing Lab of Earthquake Engineering and Structural Retrofit.

\section{References}

[1] S.-Z. Shen and X.-D. Zhi, "Failure mechanism of reticular shells subjected to dynamic actions," China Civil Engineering Journal, vol. 38, no. 1, pp. 11-20, 2005.

[2] F. Fan, X.-D. Zhi, and S.-Z. Shen, Failure Mechanism of Reticulated Shells Under Earthquake, Science Press, Beijing, China, 2014.

[3] G.-B. Nie, Research on Spatial Hysteretic Experiment and Constitutive Equation for Element of Reticulated Shells, Harbin Institute of Technology, 2008.

[4] W.-F. Du, B.-Q. Gao, and S.-L. Dong, "Double-control criterion of dynamical strength failure for single layer latticed shells," Journal of Zhejiang University, vol. 41, no. 11, pp. 1916-1926, 2007.

[5] Q.-L. Zhang and U. Peil, "Stability analysis of elastics structures under arbitrary excitation," China Civil Engineering Journal, vol. 31, no. 1, pp. 26-32, 1998.

[6] Y. Hiyama, H. Takashima, T. lijima, and S. Kato, "Buckling behavior of aluminum ball jointed single layered reticular domes," International Journal of Space Structures, vol. 15, no. 2, pp. 81-94, 2000.

[7] Z.-X. Li, Z.-Y. Shen, C.-G. Deng et al., "Research on the dynamic stability of steel reticulated shells via shaking table test," Journal of Experimental Mechanics, vol. 14, no. 4, pp. 484-491, 1999.

[8] J. Ye and R. Pan, "Shaking table test on collapse process of singlelayer spherical shells," Journal of Building Structures, vol. 34, no. 4, pp. 81-90, 2013.
[9] G. Nie, F. Fan, X. Zhi, and J. Dai, "Shaking table test on failure mechanism of single-layer reticulated dome," China Civil Engineering Journal, vol. 46, no. 10, pp. 17-25, 2013.

[10] G.-B. Nie, F. Fan, X.-D. Zhi, and J.-W. Dai, "Dynamic characteristics test of a single-layer reticulated dome model," Journal of Vibration and Shock, vol. 32, no. 22, pp. 200-204, 2013.

[11] Y.-G. Zhang, P.-F. Ba, J.-Z. Wu, and W.-J. Zhang, "Damage evolution and bearing capacity analysis of single layer spherical shell based on aging," Journal of Tianjin University (Science and Technology), vol. 47, supplement 7, pp. 74-78, 2014. 


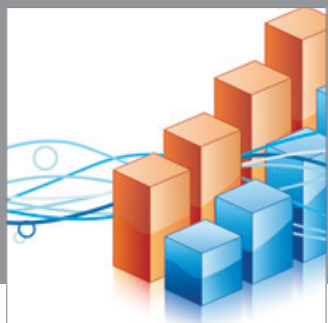

Advances in

Operations Research

mansans

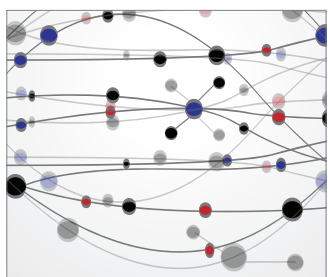

The Scientific World Journal
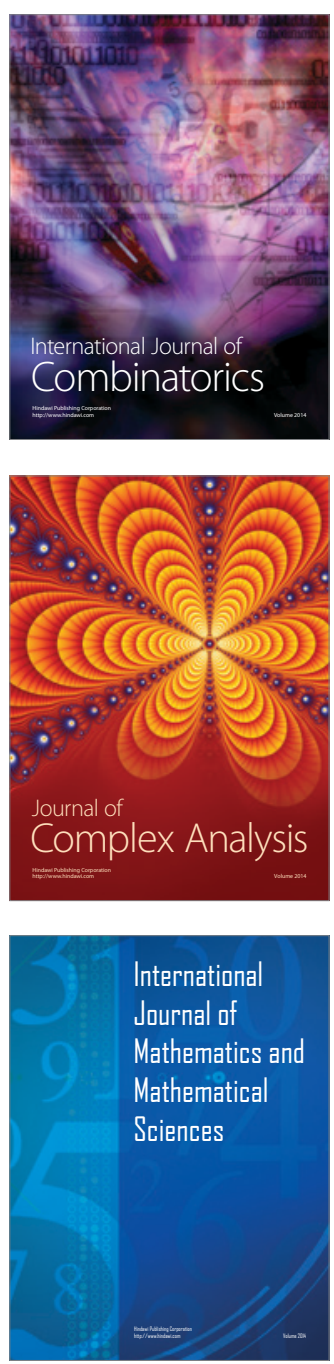
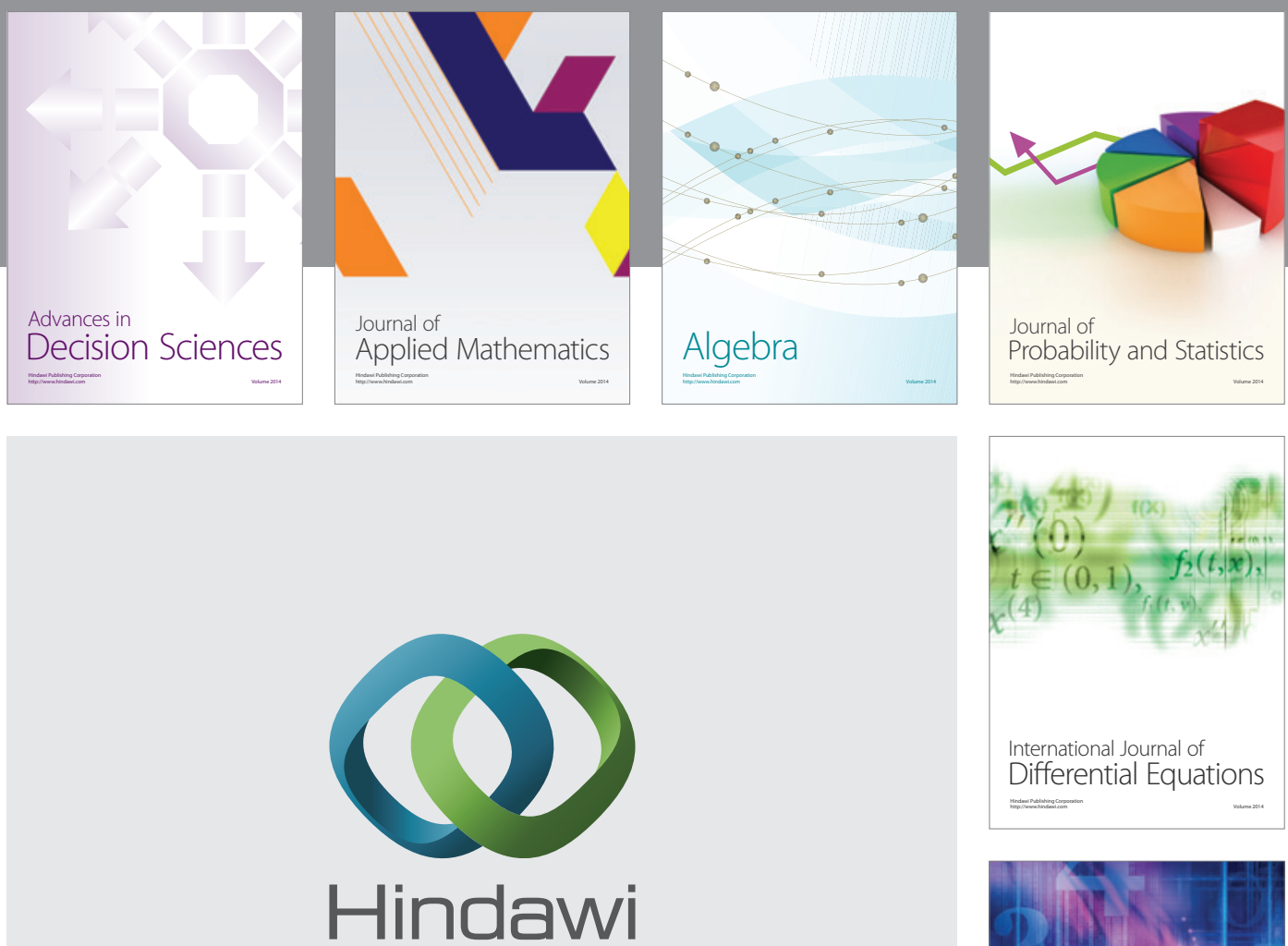

Submit your manuscripts at http://www.hindawi.com
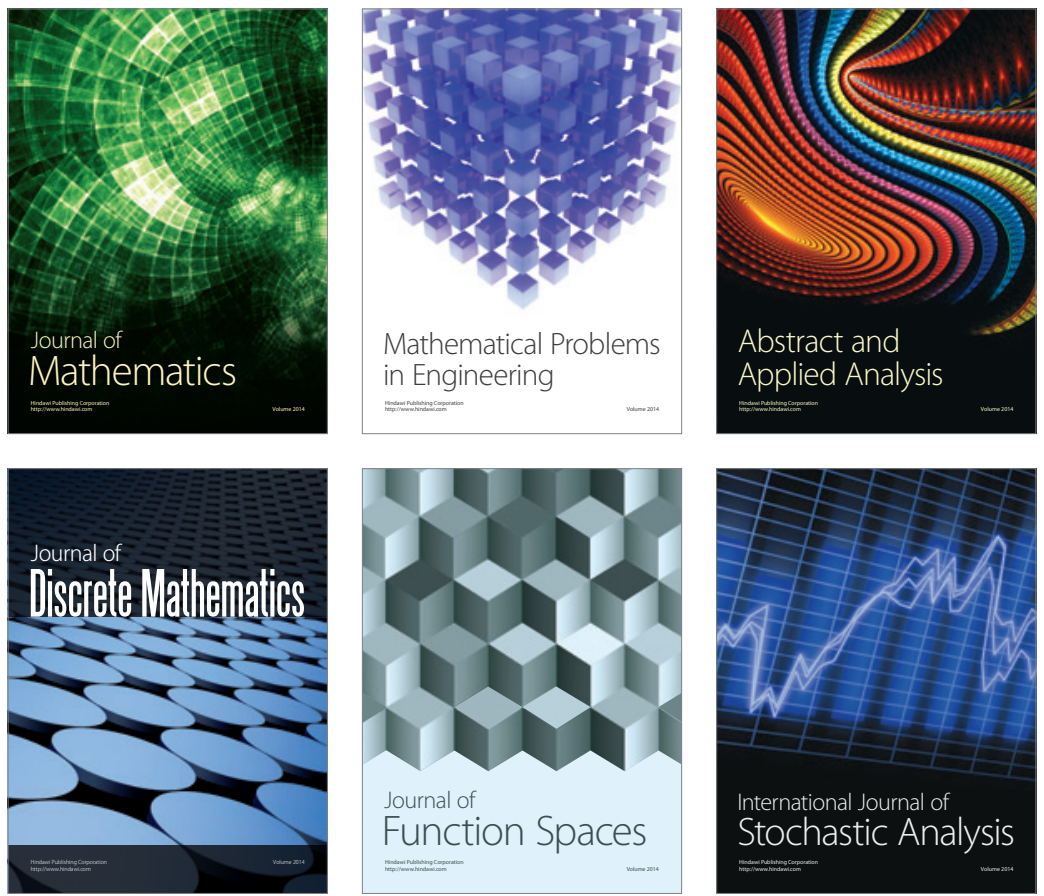

Journal of

Function Spaces

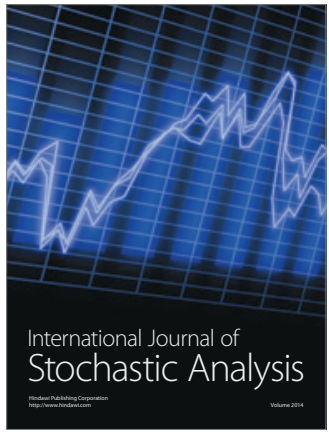

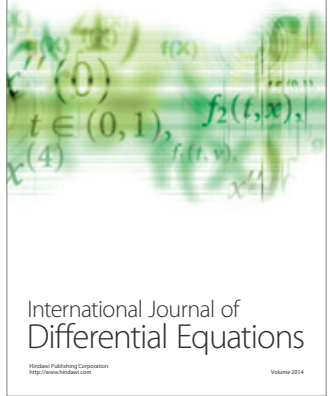
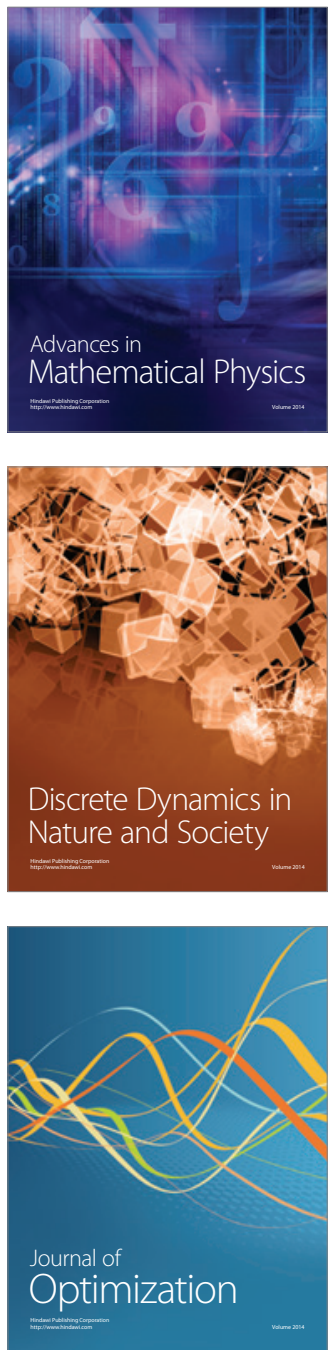